\title{
Freshmen and Senior Teaching Science and Mathematics Students' Proving Patterns and Conceptualizations of the Nature and Role of Proof in School Mathematics
}

\author{
Yeşim İmamoğlu and Ayşenur Yontar Toğrol \\ Boğaziçi University, Turkey
}

\begin{abstract}
In light of the current studies and the Turkish high school curriculum that emphasizes the importance of proof, justification and reasoning in mathematics education; this study aims to investigate freshmen and senior teaching science and mathematics students' a) conceptualizations regarding the nature and role of proof in school mathematics; b) reasoning patterns that emerge while proving mathematical statements. Data was collected in two sessions from freshmen and senior students during lecture hours, and analyzed both qualitatively and quantitatively. This is a pilot study of an ongoing research and the results are also being used for instrument development purposes.
\end{abstract}

\section{Introduction}

Recent curriculum changes in Turkey are based on constructive perspective, and highlight the importance of active, meaningful learning. In the new mathematics curriculum, conceptual learning is the basic approach. The curriculum states that the students are expected to engage in mathematics actively, learn how to solve problems, share, explain, defend their solutions and thoughts, and find relations within mathematics as well as between mathematics and other subjects [1].

General aims of the (high school) mathematics curriculum include that students will be able to: reason deductively and inductively, express their mathematical thinking and reasoning while solving problems, and use mathematical language and symbols correctly in order to communicate their mathematical thinking.

Regarding this focus on mathematical proof, this pilot study aims to investigate both freshmen and senior teaching science and mathematics students' beliefs and attitudes towards proof as well as their reasoning patterns that emerge while proving mathematical statements. Another aim of the study is to develop instrument for a larger study, hence the results of data analysis are also considered in this regard.

\section{Literature Review}

In recent views, mathematical proof is considered as "an argument needed to validate a statement, an argument that may assume several different forms as long as it is convincing" [2]. Selden \& Selden [3] consider proofs as "texts that establish the truth of theorems" and define validation of proofs as "readings of, and reflections on proofs to determine their correctness". Proving involves "constructing a deductive argument using valid rules of inference, axioms, definitions and previously proven conclusions" [4].

Proof process includes both inductive and deductive reasoning. Reasoning is a type of thinking that involves inference; the process where "one proposition (conclusion) is arrived at and accepted on the basis of other propositions (premises) that were originally accepted" [5]. In deductive reasoning inference process leads from general to particular and premises provide necessary evidence for the truth of the conclusions. In inductive reasoning, inference process leads from particular to general, and premises provide probable, but not necessary evidence for conclusions [5], [6].

An argument, in logic, is defined as "a sequence of sentences or propositions of which one (conclusion) is said to follow from others (premises), and the premises are said to provide evidence for the truth of the conclusion" [5]. A deductive argument is valid (correct) when it is impossible to have true premises and a false conclusion in the argument. Inductive arguments cannot be assigned as valid or invalid because the premises are only probable evidence for the conclusion.

Forms of reasoning such as pragmatic reasoning (based on context), statistical reasoning (based on probability) and modal reasoning (based on possibilities and necessities) involve inductive 
inferences [5]. While the final formal mathematical proof is based on deductive arguments, in the process of conjecturing or arriving at a solution (before the formal proof is given) mathematicians use inductive reasoning as well. Lakatos [7], [8] recognizes three stages of mathematical reasoning used in proofs:

First, mathematicians use induction to discover conjectures worth trying to prove. At the next stage, they develop and criticize informal proofs of their conjectures. Finally, they formalize the informal theory so that theorems are deducible by formal transformations of the axioms. According to Lakatos mathematics grows through continuous "improvement of guesses by speculation and criticism, by the logic of proof and refutation" [7]. Hence proof is never finished; its improvement and gaining acceptance depends on negotiation of meaning, which is a social process.

Sherry [9] distinguishes between quasi-empirical reasoning and quasi-experiment (or thought experiment). Quasi-empirical method in mathematics is "analogous to the methods of the physical sciences except that the singular statements which are 'generalized by induction', used to test 'theories', etc. are themselves the product of proof or calculation rather than being observation reports" [9]. It infers a theorem from a variety of singular statements. In Lakatosian quasi-experiment, the argument is in terms of a single case and the generality of the result derives from a routine supposedly applicable to any case. It infers a theorem "from the result of applying an empirical procedure to an object treated as an instance of exact concepts" [9].

Constructing proofs is inherent to making mathematics and it is an important topic in mathematics education research [10]. Miyazaki [11] demonstrates levels of proof of students in lower secondary school mathematics. Miyazaki first mentions three levels of proof theoretically established by Balacheff, on the basis of three poles (nature of conceptions, formulation, and validation). These levels, given in the order of lowest to the highest, are as follows [11], [12]:

- Pragmatic proofs: "Those having resource to actual action or showings". Types of pragmatic proofs are naïve empiricism and crucial experiment.

- Intellectual proofs: "Those which do not involve action and rest on formulations of the properties in question and relations between them". Types of intellectual proofs are generic example and thought experiment.

- Demonstration: "Requires a specific status of knowledge which must be organized in a theory and recognized as such in community; the validity of definitions, theorems and deductive rules is socially shared."

Levels of proof Miyazaki established on the basis of Balacheff's ideas are steps from inductive proof to algebraic demonstration. In this context, demonstration refers to "human activities to reason a proposition from assumptions deductively and to present the reasoning with formal language". The objective to learn demonstration is given as follows: "A student can show others the reason why a proposition is true for himself or herself'. Prerequisites to achieve this objective is specified in terms of content (what it shows), and representation (how does it show). In terms of content, the students need to deduce the proposition from assumptions that are true for themselves. In terms representation, they need to use a language called functional language of demonstration which consists of:

- Symbols and rules of their arrangement to represent objects, their properties, and relations between them.

- Terms and rules of their arrangement to represent a proposition

- Sentences and rules of their arrangement and abbreviation to represent a chain of propositions.

Miyazaki uses thee axes to establish the levels of proof: Contents, representation and students' thinking. There are two categories in each axis. Content consists of 'inductive reasoning' and 'deductive reasoning'; representation consists of 'functional language of demonstration' and 'language other than functional language of demonstration, drawings, and/or manipulable objects'; and students thinking is divided into 'concrete operations' and 'formal operations'. Combining two categories in the first two axes, four basic levels are established, as shown in table1:

Table 1. Proof levels

\begin{tabular}{|l|l|l|}
\hline $\begin{array}{c}\text { Contents } \\
\text { Representation }\end{array}$ & $\begin{array}{l}\text { Inductive } \\
\text { reasoning }\end{array}$ & $\begin{array}{l}\text { Deductive } \\
\text { reasoning }\end{array}$ \\
\hline $\begin{array}{l}\text { Functional language of } \\
\text { demonstration }\end{array}$ & Proof $D$ & Proof $A$ \\
\hline $\begin{array}{l}\text { Other language, } \\
\text { drawings, and/or } \\
\text { manipulable objects }\end{array}$ & Proof $C$ & Proof $B$ \\
\hline
\end{tabular}

\section{Research Questions}

In order to reach the aims of the study, the following research questions were put forward:

- What are freshmen/senior teaching science and mathematics students' conceptions about the nature and role of proof in school mathematics?

- What are the reasoning patterns that freshmen/senior teaching science and mathematics 
students use when they are asked to prove mathematical statements?

These questions were answered by analyzing data from freshmen and senior students both qualitatively and quantitatively. The results will help to understand prospective mathematics and science teachers' reasoning patterns and beliefs about proof in school mathematics.

\section{Sample and Data Collection}

Data was collected in two sessions from freshmen and senior mathematics and science education students. For the first session, 40 freshmen primary education science and mathematics teaching students participated in the study. These students were in the middle of the second semester of the program; therefore they had taken math courses in university. 19 senior secondary school science and mathematics students participated the second session and most of them were about to graduate at the end of that semester. The participants were asked to prove some mathematical statements (first part of the instrument) and then asked about their beliefs and thoughts about mathematical proof (second part of the instrument). For the freshmen students, mathematical statements in the first part of the instrument were divided into two groups, so that it could be completed in a shorter period of time. Second part of the instrument was identical in both sessions. Instruments were conducted in paper-pencil form. Both sessions took place within lecture hours. The lecturers and the researcher were present during these sessions.

\section{Instrument and Data Analysis}

The instrument was developed in two parts. First part consists of mathematical statements that the participants are asked to prove. Some of these statements are as follows:

- Prove that if the square of a natural number is even, then that natural number is even.

- Prove or disprove: Given any three consecutive integers, one of them will be divisible by three.

- Prove or disprove: The equality $1+3+5+\ldots+$ $2 n-1=n^{2}$ is true for all integers $n \geq 1$.

- $\quad$ Prove that, in a party of $n$ people $(\mathrm{n} \geq 2)$, there are at least two people who have the same number of friends.

The following were considered during the development of items in the instrument:

Literature: Instruments used in the studies that investigate participants' mathematical reasoning and proof techniques were examined [3], [4], [11], [13], [14], [15], [16].

Books on mathematical proof: Typical examples that can be found in books about methods of mathematical proof [17], [18], [19] were considered in item development.

Mathematical Content: Content knowledge required for the items were aimed to be kept at minimum, so that the participants' reasoning is not obstructed by the lack of knowledge in a certain mathematical subject.

Curriculum: Content covered by the items is included in the high school curriculum [1]: properties of natural numbers and integers, divisibility (grade 9, subject: algebra/numbers). All types of proof methods mentioned in the curriculum for grade 9, subject of logic [1] are covered by the items. No other proof method is needed, though may be used by the participant.

Alternative solutions: All items can be proved in several ways using alternative proof methods.

Expert opinion: Opinions and suggestions of several people including a high school teacher, two instructors from department of mathematics, an instructor from teaching mathematics department, a graduate student in teaching mathematics were taken into consideration.

Difficulty level: Items that have different levels of difficulty have been selected in order to ensure a more accurate idea about participants' reasoning skills.

Responses to mathematical statements were analyzed qualitatively in order to see the type of reasonings and proof techniques the students used.

Second part consists of items asking about thoughts, beliefs, and participants' background about mathematical proof. In this part, data was collected by a Likert type scale consisting of 16 items and one open ended question: "What does mathematical proof mean to you? Explain briefly". Response categories for the scale were from strongly disagree to strongly agree scored as follows: Strongly Disagree (1), Disagree (2), Neutral (3), Agree (4), and Strongly Agree (5). Items reflecting negative attitude were scored in reverse order. For the open ended item in the attitude scale, the responses were analyzed qualitatively to form categories.

\section{Results}

\subsection{Attitude scale}

Cronbach alpha of the attitude scale was moderately reliable $(\alpha=.60)$. Bartlett's test of sphericity $(p=.000<.05)$ indicated high correlation among items in the scale. Thus, factor analysis over 16 items were performed. As a result, five factors were extracted, four of which were interpretable due to the internal consistency condition (see Table 2). 
Table 2. Factor loadings of the Attitude Scale

\begin{tabular}{|c|c|c|c|c|c|}
\hline \multirow{2}{*}{ Item } & \multicolumn{5}{|c|}{ Component } \\
\hline & 1 & 2 & 3 & 4 & 5 \\
\hline Att12 & ,807 &,- 130 & ,064 &,- 011 &,- 068 \\
\hline Att11 & ,807 & , 111 &,- 012 &,- 089 &,- 053 \\
\hline Att10 & ,703 &,- 061 &,- 047 &, 024 &, 032 \\
\hline Att9 & ,655 &,- 010 & , 120 & ,458 & 2,060 \\
\hline Att6 & ,067 & ,831 & , 185 &, 039 &,- 033 \\
\hline Att5 & ,060 & ,811 & , 135 &, 052 &,- 054 \\
\hline Att8 &,- 212 & 675 & ,040 &,- 072 & ,076 \\
\hline Att4 &,- 325 &,- 048 & ,722 &,- 027 & , 166 \\
\hline Att15 & , 171 & ,259 & 645 &,- 223 &,- 073 \\
\hline Att16 & ,075 & ,063 & ,574 & 472 & $\overline{0}, 006$ \\
\hline Att1 &,- 071 & ,485 & ,517 &,- 157 & ,382 \\
\hline Att2 & ,084 &, 196 & ,453 &, 101 &,- 073 \\
\hline Att3 &,- 028 & ,003 &,- 056 & ,883 & $\begin{array}{r}3,91 \\
\text { E-005 }\end{array}$ \\
\hline Att14 & 279 &,- 324 & , 150 & ,092 & ,719 \\
\hline Att13 &,- 149 &, 114 &,- 451 &, 154 & ,647 \\
\hline Att7 &,- 208 & ,242 &, 113 &,- 313 & ,508 \\
\hline
\end{tabular}

Means, standard deviations and reliability coefficients of the components, as well as the corresponding item numbers are given in Table 3.

Table 3. Mean, standard deviation and reliability coefficients of the extracted factors

\begin{tabular}{|l|l|l|l|l|}
\hline & Mean & $\begin{array}{l}\text { Standard } \\
\text { Deviation }\end{array}$ & $\begin{array}{l}\text { Cronbach } \\
\text { Alpha }\end{array}$ & $\begin{array}{l}\text { Contributing } \\
\text { Items }\end{array}$ \\
\hline $\mathbf{1}$ & 2.73 & .92 & .76 & $9,10,11,12$ \\
\hline $\mathbf{2}$ & 3.25 & .81 & .73 & $5,6,8$ \\
\hline $\mathbf{3}$ & 3.35 & .64 & .63 & $1,2,4,15,16$ \\
\hline $\mathbf{4}$ & 3.63 & .70 & .31 & $7,13,14$ \\
\hline
\end{tabular}

First component consists of items about background information; how much were the students exposed to the concept of proof in high school. Contributing items are as follows (the test was originally administered in Turkish):

- In our high school, proofs were part of mathematics lessons

- In high school, I was expected to do some simple mathematical proofs during the lessons or in the exams

- Our high school teacher never talked about the importance of proof

- Our high school mathematics teacher was encouraging us to do proofs

The items in the second component are about the importance usage of proof in high school mathematics:
- Incorporating proof in high school mathematics may make it difficult for the students

- Proofs should be used when introducing concepts in high school mathematics

- A high school student should be expected to do proofs in mathematics lessons

Third component consists of items about students' personal beliefs and experiences regarding proof:

- When I see a mathematical statement (theorem, property, equality etc.) I study its proof to understand the concept.

- In order to decide whether a mathematical statement is true or false, I need to check whether it holds for all possible cases

- Mathematical proofs are usually confusing.

- I feel I am competent in doing mathematical proofs

- I do not feel confident in doing mathematical proofs

In the last component, there are items about the differences between high school and university:

- It is enough for a high school student to know the applications of a mathematical theorem or property

- I think that mathematics lectures in university will be thought in a different style than in high school

- I think that the knowledge and skills I gained in high school will be helpful to me in mathematics lectures in the university

The following item did not fit in any component, therefore discarded:

- I use examples to explain a mathematical statement to others (like to a student or a friend)

Attitude scale also included the open ended question: "What does mathematical proof mean to you? Explain briefly". Response rates are given in Table 4 below:

Table 4. Response rates to open ended item

\begin{tabular}{|l|l|l|l|l|}
\hline \multicolumn{2}{|l|}{ Grade } & Respond & N & \% \\
\hline \multirow{3}{*}{ Freshmen } & Science & 6 & 12 & 50 \\
\cline { 2 - 5 } & Math & 9 & 26 & 34,6 \\
\hline Seniors & 11 & 19 & 57,9 \\
\hline Total & $\mathbf{2 5}$ & $\mathbf{5 7}$ & $\mathbf{4 3 , 9}$ \\
\hline
\end{tabular}

The following categories emerged from the responses:

Definition of proof:

- Asserting that the statement is true for all cases. Everyone should reach the same conclusion under the same conditions.

- Mathematics is the language of science, explains science with its own system. 
- Tool for convincing the students the theorem is valid. Proof is the point where science convinces us.

- System of logical relationships that helps us understand when and why a statement is true.

- Consistent within itself, not contradictory. Uses of proof: We use proof to

- Achieve deeper, better understanding, to prevent route learning.

- Learn to use information better, learn how to use concepts.

- Prepare for mathematics courses we'll take in university. Gains of proof:

- Broadens our views and perceptions.

- Help us to think.

- Increases interest to the topic, exciting, enjoyable, it is like solving a puzzle.

Use of proof in high school mathematics:

- We cannot teach everything using proof. Using proof in every topic can be discouraging.

- Teaching some proofs can be difficult and not useful for students who are under certain level.

- In some topics teaching without proof is impossible.

- Proof should be thought in high school, otherwise we cannot learn properly in university.

- Proof is not the only way to teach/learn.

- If the students do not understand where a theorem comes from, then it will be difficult for them to apply it, they will just memorize.

\subsection{Proof of mathematical statements}

In this section the students were asked to prove some mathematical statements. There were ten items in total asked to three groups. Freshmen students were divided into two groups and asked different items (group 1A and group 1B). Group 2 refers to senior students. Each item was asked to at least two groups: For group $1 \mathrm{~A}$ and $1 \mathrm{~B}$, six questions were asked and they were expected to answer four $(3+$ Choose 1 from $3)$. Students in group 2 were asked all ten items, expected to answer $6(5+$ Choose 1 from 5). Each proof was given a grade between 0 and 4 . Grade 0 was given to proofs that were false; grade 4 was given to full proofs with sufficient explanation. Frequencies of grades for items asked to the three groups are shown in tables 5, 6, and 7 below.

Table 5. Frequencies of grades, group $1 \mathrm{~A}$

\begin{tabular}{|l|l|l|l|l|l|l|l|}
\hline Item & $\mathbf{N}$ & Attempted & $\mathbf{0}$ & $\mathbf{1}$ & $\mathbf{2}$ & $\mathbf{3}$ & $\mathbf{4}$ \\
\hline $\mathbf{2}$ & 15 & 9 & 0 & 1 & 2 & 1 & 5 \\
\hline $\mathbf{4}$ & 15 & 10 & 0 & 0 & 3 & 2 & 5 \\
\hline $\mathbf{5}$ & 15 & 4 & 0 & 3 & 0 & 1 & 0 \\
\hline
\end{tabular}

\begin{tabular}{|l|l|l|l|l|l|l|l|}
\hline $\mathbf{6}$ & 15 & 4 & 0 & 2 & 0 & 0 & 2 \\
\hline $\mathbf{9}$ & 15 & 7 & 0 & 2 & 0 & 4 & 1 \\
\hline $\mathbf{1 0}$ & 15 & 0 & 0 & 1 & 0 & 0 & 5 \\
\hline
\end{tabular}

Table 6. Frequencies of grades, group 1B

\begin{tabular}{|l|l|l|l|l|l|l|l|}
\hline Item & $\mathbf{N}$ & Attempted & $\mathbf{0}$ & $\mathbf{1}$ & $\mathbf{2}$ & $\mathbf{3}$ & $\mathbf{4}$ \\
\hline $\mathbf{1}$ & 25 & 24 & 6 & 2 & 2 & 4 & 10 \\
\hline $\mathbf{3}$ & 25 & 17 & 6 & 4 & 5 & 1 & 1 \\
\hline $\mathbf{4}$ & 25 & 20 & 0 & 0 & 3 & 3 & 14 \\
\hline $\mathbf{6}$ & 25 & 10 & 1 & 4 & 0 & 0 & 5 \\
\hline $\mathbf{7}$ & 25 & 3 & 0 & 3 & 0 & 0 & 0 \\
\hline $\mathbf{8}$ & 25 & 4 & 0 & 1 & 3 & 0 & 0 \\
\hline
\end{tabular}

Table 7. Frequencies of grades, group 2

\begin{tabular}{|l|l|l|l|l|l|l|l|}
\hline Item & $\mathbf{N}$ & Attempted & $\mathbf{0}$ & $\mathbf{1}$ & $\mathbf{2}$ & $\mathbf{3}$ & $\mathbf{4}$ \\
\hline $\mathbf{1}$ & 19 & 18 & 5 & 1 & 0 & 2 & 10 \\
\hline $\mathbf{2}$ & 19 & 17 & 1 & 0 & 0 & 1 & 15 \\
\hline $\mathbf{3}$ & 19 & 17 & 0 & 0 & 0 & 2 & 15 \\
\hline $\mathbf{4}$ & 19 & 18 & 0 & 0 & 0 & 3 & 15 \\
\hline $\mathbf{5}$ & 19 & 16 & 4 & 5 & 0 & 6 & 1 \\
\hline $\mathbf{6}$ & 19 & 4 & 0 & 2 & 0 & 0 & 2 \\
\hline $\mathbf{7}$ & 19 & 0 & 0 & 0 & 0 & 0 & 0 \\
\hline $\mathbf{8}$ & 19 & 3 & 0 & 2 & 0 & 0 & 1 \\
\hline $\mathbf{9}$ & 19 & 9 & 0 & 0 & 0 & 0 & 9 \\
\hline $\mathbf{1 0}$ & 19 & 6 & 0 & 0 & 0 & 1 & 5 \\
\hline
\end{tabular}

Categories of proofs given to the items were as follows:

Item 1: "If the square of a natural number is even, then that number must be even."

Proof 1A: If $n$ was odd, then its square would be odd. (Proof by contrapositive)

Proof 1B: If $n$ is even, then its square will be even. (This proofs the converse of the given statement; not equivalent.)

Proof1C: Assume that $\mathrm{n}$ is odd but $\mathrm{n}^{2}$ is even. If $\mathrm{n}$ is odd then $\mathrm{n}^{2}$ is be odd. This contradicts our assumption. (Proof by contradiction)

Proof1D: Direct proof: Assume $\mathrm{n}^{2}$ is even ...then $\mathrm{n}$ must be even.

Proof 1E: The square of an even number is even, the square of an odd number is odd. Hence, if the square of a number is even, then that number should be even. (Proof by cases)

Proof 1G: Giving numerical examples without generalization.

Proof 1F: There is no meaningful argument.

NA: Not attempted.

Most used (valid) proof was proof by contradiction in both groups. Commonly made mistakes were proving that the square of an even number is even, and showing the statement is true by giving numerical examples without generalization. Frequencies of the proof types are given in table 8 . 
Table 8. Proof types by groups, item 1

\begin{tabular}{|l|l|r|r|r|r|r|r|r|r|r|}
\hline \multicolumn{2}{|r|}{ Type } & NA & A & B & C & D & E & F & G & T \\
\hline Group & 1B & 1 & 9 & 6 & 1 & 2 & 3 & 0 & 3 & 25 \\
\hline & $\mathbf{2}$ & 1 & 5 & 4 & 3 & 4 & 1 & 1 & 0 & 19 \\
\hline Total & $\mathbf{2}$ & $\mathbf{1 4}$ & $\mathbf{1 0}$ & $\mathbf{4}$ & $\mathbf{6}$ & $\mathbf{4}$ & $\mathbf{1}$ & $\mathbf{3}$ & $\mathbf{4 4}$ \\
\hline
\end{tabular}

Item 2: True or false: "The equality $1+3+5+\ldots+$ $2 n-1=n^{2}$ is true for all integers $n \geq 1$."

Proof 2_A: Using Gauss' method (writing the same sum in reverse and adding up the terms).

Proof 2_B: By mathematical induction.

Proof 2_C: Using the equality

$1+2+3+\ldots+n=n(n+1) / 2$.

Proof F: There is no meaningful argument.

NA: Not attempted.

Most participants in both groups attempted proof by induction. Common mistakes made here were not showing the basis step in induction (mostly seen in group 1) and calculation errors. Frequencies of the proof types are given in table 9.

Table 9. Proof types by groups, item 2

\begin{tabular}{|l|l|r|r|r|r|r|r|}
\hline \multicolumn{2}{|r|}{ Type } & NA & A & B & C & F & T \\
\hline Groups & $\mathbf{1 A}$ & 8 & 0 & 7 & 0 & 0 & 15 \\
\hline & $\mathbf{2}$ & 1 & 2 & 14 & 1 & 1 & 19 \\
\hline Total & & $\mathbf{9}$ & $\mathbf{2}$ & $\mathbf{2 1}$ & $\mathbf{1}$ & $\mathbf{1}$ & $\mathbf{3 4}$ \\
\hline
\end{tabular}

Item3: True or false: "Given any three consecutive integers, one of them is divided by 3 ."

Proof 3_A: Direct proof: Explanations such as: When we group the numbers by three, there is a number in each group that is divisible by 3 .

Proof 3_B: Let a, a+1, $\mathrm{a}+2$ be three consecutive integers. If $\mathrm{a}=3 \mathrm{k}$ then $\mathrm{a}$ is divisible by 3 . If $\mathrm{a}=3 \mathrm{k}+1$ then $a+2$ is divisible by 3 . If $a=3 k+2$ then $a+1$ is divisible by 3 in any case, one of them will be divisible by 3 (proof by cases).

Proof 3_C: Showing that the sum is divisible by 3 . (This does not prove the statement is true: if the sum of three numbers is divisible by 3 , it cannot be concluded that one of them is divisible by 3 .)

Proof 3_D: Giving counter-example to show that the statement is false: "0 is not divisible by 3 " (the statement is not false, the counter-example is not valid because 0 is divisible by 3 ).

Proof 3_E: Giving numerical examples without generalization.

Proof 3_G: Using the divisibility rule: a number is divisible by 3 , if and only if the sum of its digits is divisible by 3 .

Proof F: There is no meaningful argument.

NA: Not attempted.

Most common proof for this item was proof by cases (using remainders). Most participants in group1 did not attempt this item. All participants who attempted this item in group 2 used proof by cases. Common mistakes were; showing the statement is false by claiming that 0 is not divisible by 3 , showing their sum is divisible by 3 , showing the statement is true by giving numerical examples without generalization. Frequencies of the proof types are given in table 10 .

Table 10: Proof types by groups, item 3

\begin{tabular}{|l|l|r|r|r|r|r|r|r|r|}
\hline \multicolumn{2}{|c|}{ Type } & NA & A & B & C & D & E & G & T \\
\hline Group & $\mathbf{1 B}$ & 10 & 2 & 3 & 2 & 2 & 5 & 1 & $\mathbf{2 5}$ \\
\cline { 2 - 10 } & $\mathbf{2}$ & 2 & 0 & 17 & 0 & 0 & 0 & 0 & $\mathbf{1 9}$ \\
\hline Total & $\mathbf{1 2}$ & $\mathbf{2}$ & $\mathbf{2 0}$ & $\mathbf{2}$ & $\mathbf{2}$ & $\mathbf{5}$ & $\mathbf{1}$ & $\mathbf{4 4}$ \\
\hline
\end{tabular}

Item 4: True or false: “(a) $n^{2}+(n+1)^{2}=(n+2)^{2}$ is true for all natural numbers

(b) $n^{2}+(n+1)^{2}=(n+2)^{2}$ is false for all natural numbers"

Proof 4_A Solve for $\mathrm{n}: \mathrm{n}=-1, \mathrm{n}=3$; the statement is true for only the natural number 3 .

Proof 4_B By giving counter-examples

Proof 4_C By giving counter-example for part a, concluding that part $\mathbf{b}$ must be true.

NA: not attempted

This was a common item that was asked to all groups, and most correctly answered item. Two different proofs were given to this statement: Solving for $n$ to find for which values of $n$ this equality is true and giving counter examples for both statements. Frequencies of the proof types are given in table 11.

Table 11. Proof types by groups, item 4

\begin{tabular}{|l|r|r|r|r|r|r|}
\hline \multicolumn{2}{|c|}{ Type } & NA & A & B & C & T \\
\hline Group & $\mathbf{1}$ & 9 & 18 & 12 & 1 & $\mathbf{4 0}$ \\
\cline { 2 - 7 } & $\mathbf{2}$ & 1 & 3 & 15 & 0 & $\mathbf{1 9}$ \\
\hline Total & & $\mathbf{1 0}$ & $\mathbf{2 1}$ & $\mathbf{2 7}$ & $\mathbf{1}$ & $\mathbf{5 9}$ \\
\hline
\end{tabular}

Item 5: "In a party of $\mathrm{n}$ people $(\mathrm{n} \geq 2)$, there exists at least two people who has the same number of friends."

Proof 5_A: General case (pigeonhole principle).

Proof 5_B: General case (induction).

Proof 5_C: Trying possibilities for small $n$ (no generalizations).

Proof F: There is no meaningful argument.

NA: Not attempted.

This was one of the least correctly answered item in all groups. The valid solution is using the Pigeonhole Principle, but even the senior students (Group 2) had problems with this item and only a few students gave a full proof. Most students who attempted to give a proof just experimented with small values of $n$, unable to generalize. Frequencies of the proof types are given in table 12 . 
Table 12. Proof types by groups, item 5

\begin{tabular}{|l|l|r|r|r|r|r|r|}
\hline \multicolumn{2}{|r|}{ Type } & NA & A & B & C & F & T \\
\hline Group & $\mathbf{1 A}$ & 11 & 2 & 0 & 2 & 0 & $\mathbf{1 5}$ \\
\cline { 2 - 8 } & $\mathbf{2}$ & 5 & 8 & 3 & 1 & 2 & $\mathbf{1 9}$ \\
\hline Total & $\mathbf{1 6}$ & $\mathbf{1 0}$ & $\mathbf{3}$ & $\mathbf{3}$ & $\mathbf{2}$ & $\mathbf{3 4}$ \\
\hline
\end{tabular}

Item 6: Sum of the interior angles of a triangle is $180^{\circ}$. Proof 6_A: Draw a line parallel to one of the sides; showing/using without proof that one of the exterior angles is the sum of the other two interior angles.

Proof 6_B: Draw a circle around the triangle (circumcircle); $2 \alpha+2 \beta+2 \gamma=360^{\circ}, \alpha+\beta+\gamma=180^{\circ}$. Length of the arc corresponding to an interior angle is twice the size of that angle.

Proof 6_C: Draw a parallel to the base, sum of the two alternate interior angles and the third interior angle gives 180 .

Proof 6_D: Using the fact that sum of exterior angles is 360 (with or without proof).

Proof 6_E: Proving the statement for right triangles (specific case).

Proof F: There is no meaningful argument.

NA: Not attempted.

This is the second item common in all groups. Common mistakes were using rules, properties which can be proved using this statement. Frequencies for the proof types are given in table 13.

Table 13: Proof types by groups, item 6

\begin{tabular}{|l|c|r|r|r|r|r|r|r|c|}
\hline \multicolumn{2}{|c|}{ Type } & NA & A & B & C & D & E & F & T \\
\hline Group & $\mathbf{1}$ & 25 & 4 & 4 & 4 & 1 & 1 & 1 & $\mathbf{4 0}$ \\
\cline { 2 - 10 } & $\mathbf{2}$ & 15 & 1 & 1 & 2 & 0 & 0 & 0 & $\mathbf{1 9}$ \\
\hline Total & $\mathbf{4 0}$ & $\mathbf{5}$ & $\mathbf{5}$ & $\mathbf{6}$ & $\mathbf{1}$ & $\mathbf{1}$ & $\mathbf{1}$ & $\mathbf{5 9}$ \\
\hline
\end{tabular}

Item 7: Product of the slopes of two perpendicular lines on the plane is -1 .

Proof 7_A: By giving examples

This was the least chosen item in both groups, and no one gave a valid proof. Frequencies are given in table 14 .

Table 14: Proof types by groups, item 7

\begin{tabular}{|l|l|r|r|r|}
\hline \multicolumn{2}{|r|}{ Type } & NA & A & T \\
\hline Grade & $\mathbf{1 B}$ & 22 & 3 & $\mathbf{2 5}$ \\
\cline { 2 - 5 } & $\mathbf{4}$ & 19 & 0 & $\mathbf{1 9}$ \\
\hline Total & & $\mathbf{4 1}$ & $\mathbf{3}$ & $\mathbf{4 4}$ \\
\hline
\end{tabular}

Item 8: Pythagoras' theorem: In a right triangle, where $c$ is the hypotenuse, and $a$ and $b$ are the legs, the following equality holds: $a^{2}+b^{2}=c^{2}$

Proof 8_A: Using Cosine theorem: $c^{2}=a^{2}+b^{2}-2 \mathrm{ab}$ $\cos \hat{\mathrm{C}}, \hat{\mathrm{m}}(\hat{\mathrm{C}})=90^{\circ}, \cos \hat{\mathrm{C}}=0, c^{2}=a^{2}+b^{2}$

Proof 8_B: Using the equality $\sin ^{2} \alpha+\cos ^{2} \alpha=1$
Proof 8_C: Draw a square for each edge. Sum of the area of the two squares is equal to the area of the third square: $a^{2}+b^{2}=c^{2}$. (There is a diagram but no other explanation.)

Proof 8_D: Draw a square with side length $a+b$. Place four right triangles with sides $a, b$, and $c$ inside the square. In the middle, there is square with side length c. Sum of the areas is $c^{2}+a b$, which is equal to the area of the big square: $(a+b)^{2}=c^{2}+2 a b, a^{2}+b^{2}+2 a b=c^{2}+$ $2 a b ; a^{2}+b^{2}=c^{2}$.

NA: Not attempted.

Common mistakes here were using rules, properties which are actually results of this statement, hence cannot be used in the proof. Frequencies of proof types are given in table 15 .

Table 15. Proof types by groups, item 8

\begin{tabular}{|l|l|r|r|r|r|r|r|}
\hline \multicolumn{2}{|r|}{ Type } & NA & A & B & C & D & T \\
\hline Group & $\mathbf{1 B}$ & 21 & 2 & 1 & 1 & 0 & $\mathbf{2 5}$ \\
\cline { 2 - 8 } & $\mathbf{2}$ & 16 & 0 & 0 & 2 & 1 & $\mathbf{1 9}$ \\
\hline Total & & $\mathbf{3 7}$ & $\mathbf{2}$ & $\mathbf{1}$ & $\mathbf{3}$ & $\mathbf{1}$ & $\mathbf{4 4}$ \\
\hline
\end{tabular}

Item 9: Let $\mathrm{A}, \mathrm{B}$ and $\mathrm{C}$ be arbitrary sets. Then, $A \cap(B U C)=(A \cap B) U(A \cap C)$.

Proof 9_A: $\mathrm{x} \in \mathrm{A} \cap(\mathrm{B} U \mathrm{C}) \leftrightarrow \mathrm{x} \in \mathrm{A}$ and $\mathrm{x} \in \mathrm{B}$ $\mathrm{U} C \leftrightarrow \mathrm{x} \in \mathrm{A}$ and $(\mathrm{x} \in \mathrm{B}$ or $\mathrm{x} \in \mathrm{C}) \leftrightarrow(\mathrm{x} \in \mathrm{A}$ and $\mathrm{x} \in \mathrm{B})$ or $(\mathrm{x} \in \mathrm{A}$ and $\mathrm{x} \in \mathrm{C}) \leftrightarrow \mathrm{x} \in(\mathrm{A} \cap \mathrm{B})$ or $\mathrm{x}$ $\in(\mathrm{A} \cap \mathrm{C}) \leftrightarrow \mathrm{x} \in(\mathrm{A} \cap \mathrm{B}) \mathrm{U}(\mathrm{A} \cap \mathrm{C}) \leftrightarrow \mathrm{A} \cap(\mathrm{B} U$ $\mathrm{C})=(\mathrm{A} \cap \mathrm{B}) \mathrm{U}(\mathrm{A} \cap \mathrm{C})$.

Proof 9_B: Follows from distribution property. (They are asked to prove the distribution property.)

NA: Not attempted.

While most people used the correct approach in this item, students in group 1 made mistakes such as using $=$ or $\rightarrow$ (if...then) instead of $\leftrightarrow$ (if and only if). Frequencies of proof types are given in table 16 .

Table 16. Proof types by groups, item 9

\begin{tabular}{|l|l|r|r|r|r|}
\hline \multicolumn{2}{|r|}{ Type } & NA & A & B & T \\
\hline Group & $\mathbf{1 A}$ & 8 & 5 & 2 & $\mathbf{1 5}$ \\
\cline { 2 - 6 } & $\mathbf{2}$ & 10 & 9 & 0 & $\mathbf{1 9}$ \\
\hline Total & & 18 & 14 & 2 & $\mathbf{3 4}$ \\
\hline
\end{tabular}

Item 10: Let $(a, b)$ and $(c, d)$ be two points in the plane. The distance between these points is $\sqrt{(a-c)^{2}+(b-d)^{2}}$

Proof 10_A: Using Pythagoras' theorem:

$\mathrm{A}=(\mathrm{a}, \mathrm{b}), \mathrm{B}=(\mathrm{b}, \mathrm{c})$ (forms a right triangle with vertices $\mathrm{A}, \mathrm{B}$ and $\mathrm{C})$. $|A B|=\sqrt{|A C|^{2}+|C B|^{2}}=\sqrt{(b-d)^{2}+(a-c)^{2}}$

All participants who attempted this item used provided this proof, which they know well from high school. Frequencies are given in table 17. 
Table 17. Proof types by groups, item 10

\begin{tabular}{|l|l|r|r|r|}
\hline \multicolumn{2}{|r|}{ Type } & NA & A & T \\
\hline Group & $\mathbf{1 B}$ & 19 & 6 & $\mathbf{2 5}$ \\
\cline { 2 - 5 } & $\mathbf{2}$ & 13 & 6 & $\mathbf{1 9}$ \\
\hline Total & & $\mathbf{3 2}$ & $\mathbf{1 2}$ & $\mathbf{4 4}$ \\
\hline
\end{tabular}

\section{Conclusion}

\subsection{Conceptualizations regarding the nature and role of proof}

Attitude scale revealed four factors, labeled as background, importance of proof in mathematics lessons, personal beliefs and experiences about proof and differences between high school and university mathematics lessons. Among these categories, background has the lowest mean $(M=2.73, S D=.92)$. It can be said that the students were not exposed to proof too much in high school. The other categories have means higher than 3.00 . We can conclude that, in general, the students have positive attitude towards proof and they believe proof has important in mathematics classes, especially in university. Responses to the open ended item also support this conclusion. It must be kept in mind that, the freshmen students were in the middle of their second semester during data collection, they have taken mathematics courses in university that focus on mathematical proofs.

\subsection{Reasoning patterns}

While most of the participants approached the proofs using deductive reasoning and functional language of demonstration (proof type A, according to Miyazaki [11]), freshmen students still resort to giving numerical examples without generalizations (inductive reasoning) in some cases.

According to Andrew's proof error evaluation tool [19], errors participants made in the proofs can be listed as follows:

Proof structure:

- Made a false assumption (e.g. ' 0 is not divisible by 3')

- Proof type: Needed to show $\mathrm{p} \rightarrow \mathrm{q}$ but did not show directly, by contrapositive or contradiction (e.g. proving $\mathrm{q} \rightarrow \mathrm{p}$ : 'if $\mathrm{n}$ is even, then $\mathrm{n} 2$ is even')

- Proof setup: The approach taken in proving a statement will not work (e.g. using induction in item 5)

- Only gave an example to establish the truth of a mathematical statement.
Conceptual Understanding:

- Did not sufficiently justify a crucial statement (e.g. 'if $\mathrm{n}$ is odd then its square is odd')

- Included some cases but not others (which were not trivial)

This study was conducted as a part of a larger study, main purpose being instrument development. The results are mostly viewed in this regard. Some alterations and improvements were made to the instrument using these results: For the attitude scale, new items were included to increase reliability. The new version was administered to 94 freshmen students in another university in their first week of their first semester. The reliability score was high $(\alpha=.88)$. The student proofs collected from this study was used to develop a third part to the instrument; different student proofs (not necessarily correct) are given to senior students and they are asked to evaluate these proofs.

As mentioned above, importance of proof in school mathematics is emphasized both in the current high school curriculum [1] and various recent research studies [1], [2], [4], [6]. Therefore, both high school students and prospective mathematics teachers should be sufficiently competent in this regard. Participants of the ongoing study are freshmen and senior mathematics and teaching mathematics students. Data is collected from freshmen students in the first week of their first semester in university. The reason for conducting such a study to this group of students is that, they are prospective mathematics teachers and therefore important figures who will shape high school students' conceptualizations related to mathematical concepts in the future. Participants of the study are newly graduated from various high schools, and students who are about to graduate from university to become mathematics teachers and mathematicians. These characteristic give an idea about the basic tendencies of conceptualizations about proof in high school and university graduates. Therefore, the ongoing study will be an important step for understanding and comparing prospective mathematics teachers' reasoning patterns at the time of starting the program and finishing it. Clarification of these reasoning patterns will be helpful in developing instructional implications for teaching mathematics programs as well as being helpful for instructors of freshman mathematics courses in terms of showing some of the tendencies in reasoning patterns seen in high school graduates. 


\section{References}

[1] Turkish Ministry of Education, "Orta öğretim matematik $(9,10,11$ ve 12 . Sinıflar) dersi ögretim programı" (High school curriculum grades 9-12). Retrieved May 16, 2008, from http: ogm.meb.gov.tr

[2] G. Hanna, "Mathematical proof", In D. Tall (Ed.), Advanced Mathematical Thinking, Kluwer Academic Publishers, Hingham MA, 1991.

[3] A. Selden and J. Selden, "Validations of proofs considered as texts: Can undergraduates tell whether an argument proves a theorem?", Journal for Research in Mathematics Education, 34(1), 2003, pp. 4-36.

[4] A. K. Morris, "Mathematical reasoning: Adults' ability to make the inductive-deductive distinction", Cognition and Instruction, 20(1), 2002, pp. 79-118.

[5] W.F. Overton (ed.). Reasoning, necessity, and logic: Developmental perspectives, Hillsdale, N.J., L. Erlbaum Associates, 1990.

[6] A. K. Morris, "Factors affecting pre-service teachers' evaluations of the validity of students' mathematical arguments in classroom contexts", Cognition and Instruction, 25(4), 2007, pp. 479522.

[7] I. Lakatos, Proofs and refutations, Vol.2, Cambridge, Cambridge University Press, 1976.

[8] I. Lakatos, Mathematics, science and epistemology: Philosophical papers, Cambridge, Cambridge University Press, 1978.

[9] D. Sherry, "Mathematical reasoning: induction, deduction and beyond", Studies in History and Philosophy of Science, 37, 2006, pp. 489-504.

[10] G. Hanna, "Proof, explanation and exploration: An overview", Educational Studies in Mathematics, 44, 2000, pp. 5-23.

[11] M. Miyazaki, "Levels of proof in lower secondary school mathematics", Educational Studies in Mathematics, 41, 2000, pp. 47-68.

[12] N. Balaceff, "Aspects of proof in pupils' practice of school mathematics", in D. Pimm (ed.), Mathematics, teachers and children Hodder and Stoughton, London, 1988, pp. 216, 230.

[13] D. Almedia, "A survey of mathematics undergraduates' interaction with proof: some implications for mathematics education", International Journal in Mathematics Education, Science and Technology, 31(6), 2000, pp. 869-890.

[14] E. J. Knuth, "Teachers' conceptions of proof in the context of secondary school mathematics", Journal of Mathematics Teacher Education, 5, 2002, pp. 61-88.
[15] Ö. Özer and A. Arıkan, "Lise matematik derslerinde öğrencilerin ispat yapabilme düzeyleri”, Paper presented at the Congress of $V$. National Science and Mathematics Education, 16-18 September, 2002, Ankara.

[16] A. Stylianides, G. Stylianides, G.N. Philippou, 'Undergraduate students' understanding of the contraposition equivalence rule in symbolic and verbal contexts", Educational Studies in Mathematics, 55, 2004, pp. 133-162.

[17] A. Cupillari, The nuts and bolts of proofs (2nd ed.), San Diego, CA, Academic Press, 2001.

[18] J. P. D'Angelo, J. P. and D. B. West, Mathematical thinking: Problem solving and proofs (2nd ed.), Upper Saddle River, NJ, Prentice Hall, 2000 .

[19] D. Solow, How to read and do proofs (4th ed.), Hoboken, NJ, John Wiley \& Sons, Inc., 2005.

[20] L. Andrew, "Creating a proof evaluation tool for use in the grading of student generated proofs", Primus, Problems, Resources and Issues in Mathematics Undergraduate Studies, 19(5), 2009, pp.447-462. 